\title{
New improvement of Gerschgorin Radii Base on Jacobi Method for Source Number Estimation
}

\author{
Wenyang Zhao ${ }^{1+}$ and Lu Gan ${ }^{1}$ \\ ${ }^{1}$ Department of Electronic Engineering, University of Electronic Science and Technology of China, Chengdu, \\ 611731, China
}

\begin{abstract}
In this paper, a new improvement of the classical gerschgorin disk method based on Jacobi method is proposed. Results of the analysis and the computer simulations are showed that the improved method consumes less multiplications and additions. It also has the same performance of the classical gerschgorin disk method. In a word, this new approach not only can reduce the consumption of resource and time, but also has a good performance of the sources number estimation. Compared with the classical gerschgorin disk method, this new method base on Jacobi method iterative structure, is more easy to implement in FPGA and DSP.
\end{abstract}

Keywords: Gerschgorin disk estimator, Source number estimation, Jacobi method.

\section{Introduction}

The sources number estimation plays an important role in spatial spectrum estimation, which is widely used in communication, radar, and sonar etc. And most of the algorithms of spatial spectrum estimation need to know the number of sources in advance. When the estimate of sources number is not correlated with the actual number of sources number, it will cause error in the result of the spatial spectrum estimation.

There are many algorithms to get the incident signals number, such as the smoothed-rank sequence method [1], the information theoretic criterion (AIC, MDL)[2][3], the canonical correlation technique (CCT)[4] and the gerschgorin disk method (GDE)[5][6], and different algorithms have different performance in different situations. For example, in a low signal to noise ratio (SNR) environment, the smoothed-rank sequence method has a poor performance [7]. The canonical correlation technique and the gerschgorin disk method can be applied in an environment with colored noise [8], but the information theoretic criterion cannot get the correct number of sources when the noise is not white [9]. Some methods of estimating the number of sources, including the smoothed-rank sequence method and the information theoretic criterion, need to obtain the eigenvalues of the covariance matrix, and then use the eigenvalues to estimate the number of signal sources.

In the classical gerschgorin disk method, we need to make the eigenvalue decomposition firstly and then transform the covariance matrix base on the feature space. After doing these steps, we obtain the sources number by the detection rule [5]. We can see that the procedure of the classical gerschgorin disk method is complicated. So it is difficult to be implemented in FPGA or DSP. In this paper, we propose a novel improved edition of the classical gerschgorin disk method base on Jacobi method. This method is much simpler than the classical one, and also has all advantages of the classical gerschgorin disk method. In addition, it is simpler to be implemented in the FPGA and DSP than the classical gerschgorin disk method.

\section{Signal Model}

\footnotetext{
+ Corresponding author. Tel.: +8618200258607 .

E-mail address: wenyangzhao@yeah.net.
} 
Assume $N$ narrow band far-field sources $s_{k}(n)$ from distinct directions $\gamma_{k}, k=1 \sim N$, impinging on a uniform linear array (ULA) with $M(M>N)$ omnidirectional array elements. For simplicity, we assume that the array elements and the sources are in the same plane, and the element spacing $d=\lambda / 2$, where $\lambda$ is the wavelength. Then, the $M \times 1$ output vector of the array with independent snapshots can be expressed as below:

$$
X(n)=\sum_{k=1}^{N} a\left(\gamma_{k}\right) s_{k}(n)+V(n) \quad n=1,2, \cdots, L
$$

Where $X(n)=\left[\begin{array}{llll}x_{1}(n) & x_{2}(n) & \cdots & x_{M}(n)\end{array}\right]^{T} \in C^{M \times 1}$ is the output vector of the array and the $x_{i}(n)$ is the received signal of the $i$ th sensors. $V(n) \in C^{M \times 1}$ is the additive noise. $a\left(\gamma_{k}\right) \in C^{M \times 1}$ is the steering vector of the array which can describe the transfer function between the direction $\gamma_{k} \in(-\pi / 2, \pi / 2)$ and the output of the array, it can be modeled as below:

$$
a\left(\gamma_{k}\right)=\left[\begin{array}{llll}
1 & e^{-j \phi_{k}} & \cdots & e^{-j(M-1) \phi_{k}}
\end{array}\right]^{T}, \phi_{k}=2 \pi d \sin \gamma_{k} / \lambda=\pi \sin \gamma_{k}, k=1 \sim N
$$

In matrix form, we can define the source vector $S(n) \in C^{N \times 1}$ and the direction matrix $A$ as below:

$$
\begin{aligned}
& S(n)=\left[\begin{array}{llll}
s_{1}(n) & s_{2}(n) & \cdots & s_{N}(n)
\end{array}\right]^{T} \in C^{N \times 1} \\
& A=\left[\begin{array}{llll}
a\left(\gamma_{1}\right) & a\left(\gamma_{2}\right) & \cdots & a\left(\gamma_{N}\right)
\end{array}\right]=\left[\begin{array}{cccc}
1 & 1 & \cdots & 1 \\
e^{-j \phi_{1}} & e^{-j \phi_{2}} & \cdots & e^{-j \phi_{N}} \\
\vdots & \vdots & & \vdots \\
e^{-j(M-1) \phi_{1}} & e^{-j(M-1) \phi_{2}} & \cdots & e^{-j(M-1) \phi_{N}}
\end{array} \mid \in C^{M \times N}\right.
\end{aligned}
$$

then, the received data can be simplified as below:

$$
X(n)=A S(n)+V(n)
$$

\section{Gerschgorin Disk Method}

The Gerschgorin disk method is a good way to estimate the number of sources, it was proposed by Wu et al.[5] in 1995. Here, we give the calculation steps of this algorithm as below:

Step1 Calculate the covariance matrix $\hat{R}$ according to the following formula:

$$
\hat{R}=\frac{1}{L} \sum_{n=1}^{L} X(n) X^{H}(n)
$$

Step2 Calculate the feature space $\hat{U}$ of $\bar{R} \in C^{(M-1) \times(M-1)}$.

Where $\hat{R}=\left[\begin{array}{cc}\bar{R} & \hat{r} \\ \hat{r}^{H} & \hat{r}_{M M}\end{array}\right] \in C^{M \times M}, \hat{U} \hat{U}^{H}=E$, and $\bar{R}=\hat{U} \Sigma \hat{U}^{H}$.

Step3 Use the the following formula to transform the covariance matrix $\hat{R}$.

$$
\hat{R}_{T}=T^{H} \hat{R} T=\left[\begin{array}{cc}
\Sigma & \hat{U}^{H} \hat{r} \\
\hat{r}^{H} \hat{U} & \hat{r}_{M M}
\end{array}\right]=\left[\begin{array}{ccccc}
\hat{\lambda}_{1} & & & & \rho_{1} \\
& \hat{\lambda}_{2} & & & \rho_{2} \\
& & \ddots & & \vdots \\
& & & \hat{\lambda}_{M-1} & \rho_{M-1} \\
\rho_{1}^{*} & \rho_{2}^{*} & \cdots & \rho_{M-1}^{*} & \hat{r}_{M M}
\end{array}\right]
$$

Step 4 Use the following decision rule to determine the number of source.

$$
G D E(k)=r_{k}-\frac{D(L)}{M-1} \sum_{i=1}^{M-1} r_{i}>0, k=1 \sim M-1
$$

Where $D(L)$ is an adjustable factor, which is determined by the number of snapshots $L . r_{i}=\left|\rho_{i}\right|$ is the Gerschgorin radii. By detecting the first non-positive value of $G D E\left(k_{0}\right)$, we can get the number of signal sources as $N=k_{0}-1$.

\section{Improve Gerschgorin Disk Method}


As we can see, in the calculation steps of gerschgorin disk method, in order to obtain the sources number, we need to calculate the eigenvalue decomposition of matrix $\bar{R}$, and then transform the covariance matrix $\hat{R}$. It is complicated, and it is difficult to be implemented in an FPGA or DSP. Here, we will give a simple calculation method, which is based on the jacobi method.

The Jacobi method approximates the EVD iteratively as follows [10].

$$
\begin{gathered}
\Sigma=P_{t}^{H} \cdots P_{2}^{H} P_{1}^{H} \bar{R} P_{1} P_{2} \cdots P_{t} \\
\hat{U}=P_{1} P_{2} \cdots P_{k}
\end{gathered}
$$

$$
P_{k}(\rho, \theta)=\left[\begin{array}{cccc}
1 & & i_{k} & j_{k} \\
& \ddots & & \\
& & e^{j \rho / 2} \cos \theta & -e^{j \rho / 2} \sin \theta \\
& e^{-j \rho / 2} \sin \theta & e^{-j \rho / 2} \cos \theta
\end{array}\right] j_{k}
$$

where $\Sigma$ is the eigenvalues of $\bar{R}, \hat{U}$ is the feature space of $\bar{R}, P_{k}$ and is an orthonormal plane rotation by $\rho$ and $\theta$ in the plan.Then, substituting (10) into (7), we can get as below.

$$
\hat{R}_{T}=\left[\begin{array}{cc}
\hat{U}^{H} \bar{R} \hat{U} & \hat{U} \hat{r} \\
\hat{r}^{H} \hat{U} & r_{M M}
\end{array}\right]=\left[\begin{array}{cc}
\hat{U} & 0 \\
0 & 1
\end{array}\right]^{H}\left[\begin{array}{cc}
\bar{R} & \hat{r} \\
\hat{r}^{H} & \hat{r}_{M M}
\end{array}\right]\left[\begin{array}{cc}
\hat{U} & 0 \\
0 & 1
\end{array}\right]
$$

Furthermore:

$$
\hat{R}_{T}=\left[\begin{array}{cc}
P_{t} \cdots P_{1} & 0 \\
0 & 1
\end{array}\right]^{H}\left[\begin{array}{cc}
\bar{R} & \hat{r} \\
\hat{r}^{H} & \hat{r}_{M M}
\end{array}\right]\left[\begin{array}{cc}
P_{t} \cdots P_{1} & 0 \\
0 & 1
\end{array}\right]
$$

Thus, we can get a new iteration equation. It is a new way to get the matrix $\hat{R}_{T}$.

$$
\hat{R}_{T}=T_{t}^{H} \cdots T_{2}^{H} T_{1}^{H} \hat{R} T_{1} T_{2} \cdots T_{t}
$$

where $T_{k}=\left\lfloor\begin{array}{cc}P_{k} & 0 \\ 0 & 1\end{array}\right\rfloor, k=1 \sim t$, and $t$ is the number of iterations.

Here is the summary of the proposed algorithm.

Step1 calculate the covariance matrix.

$$
\hat{R}=\frac{1}{L} \sum_{n=1}^{L} X(n) X^{H}(n)
$$

Step2 transform the covariance matrix.

For $k=1$ to $t$ do

$$
\begin{aligned}
& \text { For } i=1 \text { to } M-2 \text { do } \\
& \begin{aligned}
\text { For } j & =i+1 \text { to } M-1 \text { do } \\
p & =0.5 \operatorname{angle}\left(\hat{r}_{i j}\right) \\
\theta & =0.5 \arctan \left(\frac{2\left|\hat{r}_{i j}\right|}{\hat{r}_{i i}-\hat{r}_{j j}}\right) \\
\hat{R} & =T_{k}^{H} R T_{k}
\end{aligned}
\end{aligned}
$$

End for

\section{End for}

End for

Then we can obtain transform result $\hat{R}_{T}=\hat{R}$. 
Step3 determine the source number.

$$
G D E(k)=r_{k}-\frac{D(L)}{M-1} \sum_{i=1}^{M-1} r_{i}>0, i=1 \sim M-1
$$

Where $D(L)$ is an adjustable factor, which is determined by the number of snapshots $L . r_{i}=\left|\rho_{i}\right|$ is the Gerschgorin radii. By detecting the first non-positive value of $\operatorname{GDE}\left(k_{0}\right)$, we can get the number of signal sources as $N=k_{0}-1$.

Here we analyze the differences between the classical gerschgorin disk method and our method. And then give the difference in their calculation. For comparison, we assume that we also use the jacobi method to calculate the feature space $\hat{U}$ in the classical gerschgorin disk method.

In the classical gerschgorin disk method we use the following formula to get the $\hat{R}_{T}$ :

$$
\begin{gathered}
\left\{\begin{array}{l}
\Sigma=P_{t}^{H} \cdots P_{2}^{H} P_{1}^{H} \bar{R} P_{1} P_{2} \cdots P_{t} \\
\hat{U}=P_{1} P_{2} \cdots P_{t}
\end{array}\right. \\
R_{T}=\left[\begin{array}{ll}
\hat{U}^{H} \bar{R} \hat{U} & \hat{U} \hat{r} \\
\hat{r}^{H} \hat{U}^{H} & r_{M M}
\end{array}\right\rfloor
\end{gathered}
$$

In the formula (9), It contains $4(m-1) t+4 m t$ multiplications and additions, and the formula (9) contains $2(m-1)^{3}+(m-1)^{2}$ multiplications and additions. So, the classical gerschgorin disk method contains $N_{c}=4(m-1) t+4 m t+2(m-1)^{3}+(m-1)^{2}$ multiplications and additions.

In our improved way, we get theas below. And it just contains $N_{u s}=4 m t+4 t$ multiplications and additions.

$$
\hat{R}_{T}=\left\lfloor\begin{array}{cc}
P_{t}^{H} \cdots P_{2}^{H} P_{1}^{H} R P_{1} P_{2} \cdots P_{t} & P_{1} P_{2} \cdots P_{t} \hat{r} \\
\hat{r}^{H} P_{t}^{H} \cdots P_{2}^{H} P_{1}^{H} & \hat{r}_{M M}
\end{array}\right\rfloor
$$

Table I: The Number of Multiplications And Additions

\begin{tabular}{|c|c|c|c|c|c|c|}
\hline$(M, t)$ & $(4,2)$ & $(4,4)$ & $(8,2)$ & $(8,4)$ & $(12,2)$ & $(12,4)$ \\
\hline classical method & 119 & 175 & 855 & 975 & 2967 & 3151 \\
\hdashline our method & 40 & 80 & 72 & 144 & 104 & 208 \\
\hline
\end{tabular}

We can see that our improved method can reduce the number of multiplications and additions.

\section{Simulation Result}

In the second section, we built a signal model. Therefore, considering a $M=8$ sensors symmetric uniform linear array (ULA) with the element spacing $d=0.5 \lambda$. There are three $(N=3)$ far-field sources located at $\gamma_{1}=-10^{\circ}, \quad \gamma_{2}=7^{\circ}$ and $\gamma_{3}=52^{\circ}$, and the number of snapshot is $L=512$.

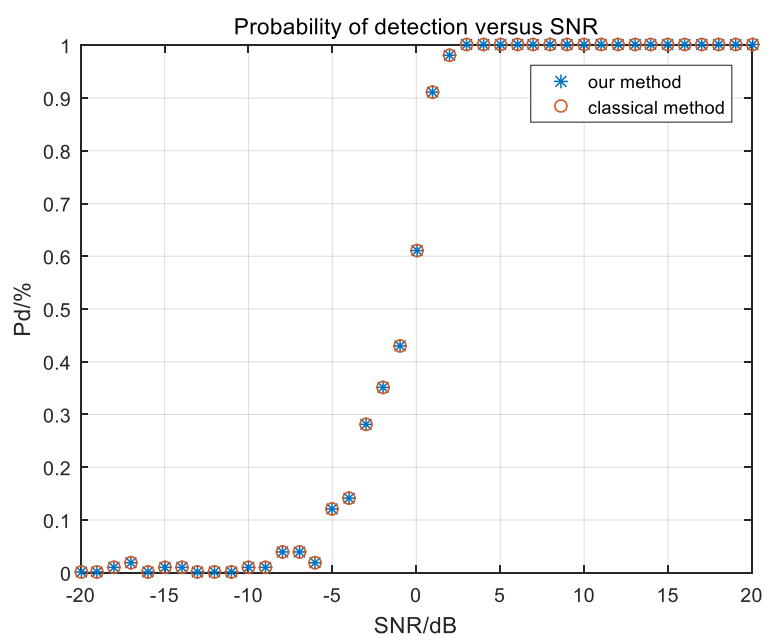

Fig. 1: Probability of detection versus SNR 
In Fig. 1, we can see that as the signal-to-noise (SNR) increases, the correct probability of detection (Pd) increases. It has a good performance when the SNR is greater than $3 \mathrm{~dB}$, and out improved method also has the same performance as the classical method.

As to the speed of calculation, we simulate by a computer with i7 6700k processor and $16 \mathrm{~g}$ ddr4 ram. In the Table 2, we can see that our improved consumes less time than the classical method.

Table II. The Consumption of Time

\begin{tabular}{|c|c|c|c|c|c|c|}
\hline$(M, t)$ & $(4,2)$ & $(4,4)$ & $(8,2)$ & $(8,4)$ & $(12,2)$ & $(12,4)$ \\
\hline classical method /ms & 9.005 & 10.144 & 12.165 & 13.347 & 15.468 & 18.509 \\
\hdashline our method /ms & 8.249 & 9.416 & 11.677 & 12.545 & 12.061 & 13.533 \\
\hline
\end{tabular}

\section{Conclusion}

In this paper, the number of sources detection method using improved gerschgorin disk criterion is proposed. Because of the poor use of the classical gerschgorin disk method in FPGA and DSP, we apply Jacobi method to simplify calculation process. And the Simulation result show that this new method also has a good performance in sources number detection.

\section{References}

[1] T. J . Shan, A . Paulray and Thomas Kailath. On Smoothed Rank Profile Tests in Eigenstructure Approach to Directions-of-Arrival Estimation. IEEE Trans. On ASSP, 1987,35(10):1377 1385.

[2] H. Akaike. A new look at the statistical model identification. IEEE Trans. On AC, 1974,19(6):716 723.

[3] G. Schwartz. Estimation the dimension of a model. Ann. Stat, 1978,6:461 464.

[4] W. Chen and J. P. Reilly. Detection of the Number of Signal in Noise with Banded Covariance Matrices. IEEE Trans. On SP, 1992, 42(5): 377 380.

[5] H. T. Wu, J. Yang and F. K. Chen. Source number estimation using transformed gerschgorin radii. IEEE Trans. On SP, 1995, 43(6): 1325 1333.

[6] H. T. Wu, J. F Yang and F. K. Chen. Source number estimator using Gerschgorin disks. IEEE International Conference on Acoustics, Speech, and Signal Processing, vol.4, pp.261-264, 1994.

[7] W. Chen, J. P. Reilly and K. M. Wong. Detection of the number of signal in noise with banded covariance matrices. IEEE Prceedings Raddar, Sonar and Navigation, vol.143, no.5, pp.289-294, 1996.

[8] H. T. Wu, J. F Yang and F K. Chen, Source number estimators using transformed Gerschgorin radii. IEEE Transactions on Signal Processing, vo1.43, no.6, pp.1325-1333, 1995.

[9] B. Nadler. Nonparametric detection of signals by information theoretic criteria: performance analysis and an improved estimator. IEEE Transactions on Signal Processing, vo1.58, no.5, pp. 2746-2756, 2002.

[10] G. E. Forsythe and P. Henrici. The cyclic Jacobi method for computing the principal values of a complex matrix. IEEE Trans, Amer. Math. Soc. 949(1960),1-23. 